\title{
PF24RT24 Plasma
}

National Cancer Institute

\section{Source}

National Cancer Institute. PF24RT24 Plasma. NCI Thesaurus. Code C133343.

Plasma Frozen within 24 Hours after Phlebotomy held at Room Temperature Up To 24 Hours after Phlebotomy (PF24RT 24). Plasma prepared from automated apheresis collections can be held at room temperature for up 24 hours after collection and then frozen at -18 degrees $C$ or colder. 